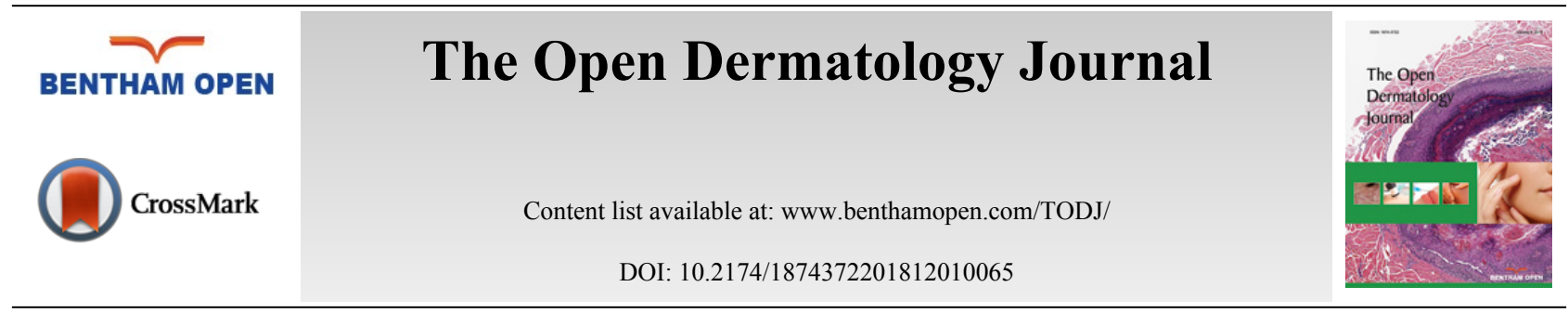

CASE REPORT

\title{
Pyoderma Gangrenosum of the Face: A Rare Presentation and a Rapid Resolution
}

\author{
Julia Shah", Lorie Gottwald, Ashley Sheskey and Craig Burkhart \\ Department of Dermatology, University of Toledo College of Medicine, Toledo, USA
}

Received: March 14, 2018

Revised: April 3, 2018

Accepted: May 21, 2018

\begin{abstract}
:
Background:

Pyoderma Gangrenosum (PG) is a disorder of neutrophil chemotaxis that often affects the lower extremities of patients with concurrent autoimmune disorders.
\end{abstract}

\section{Result and Discussion:}

Resolution of lesions typically requires a minimum of six weeks of treatment with systemic steroids. We present a unique case of multifocal PG involving the hand and face that healed after ten days of treatment with oral prednisone.

Keywords: Pyoderma Gangrenosum, Neutrophil chemotaxis, Seronegative Arthritis, MCPJ, Prednisone theraphy, IgG.

\section{INTRODUCTION}

A 51-year-old female with a history of Seronegative Arthritis presented with lesions on multiple parts of her body that had erupted over the past eighteen months. She described the lesions as large, painful papules which she would "pop" for relief. The distribution involved a new, active lesion on the right knuckle and lesions in various stages of healing on the left nostril and left cheek. She reported previous lesions on the jaw, genital mucosa, and toe. She believed the lesions were precipitated by small cuts or injuries and noted flares when she was stressed.

Physical examination revealed two erythematous, eroded papules on the left jawline (Fig. 1), a vesicle with surrounding erythema on the rim of the left nostril (Fig. 2), and a hemorrhagic, necrotic plaque on the right fifth metacarpal phalangeal joint (MCPJ) (Fig. 3).

A punch biopsy of the active lesion over the right fifth MCPJ was conducted. The patient was given mupirocin $2 \%$ topical ointment and instructed to apply it to the lesions three times daily.

The radial side of the right fifth MCPJ biopsy was submitted for immunofluorescence and stained negative for IgG, IgA, C3, fibrinogen, and albumin. The ulnar side of the biopsy sample was fixed with H\&E staining. The sample spanned from the epidermis to the subcutaneous fat. There was marked irregular epidermal hyperplasia bordering an ulcer with underlying dense neutrophilic infiltrate and an undermining edge within the dermis. There were interstitial neutrophils and crushed lymphocytes in between the collagen bundles, but there was no definite evidence of vasculitis. There was no evidence of viral inclusions, interface dermatitis, or malignancy.

The findings of an undermined edge and neutrophilic dermatosis were consistent with a diagnosis of Pyoderma Gangrenosum (PG). The patient was started on a 10 day course of $60 \mathrm{mg}$ oral Prednisone therapy. * Address correspondence to this author at the Department of Dermatology, University of Toledo College of Medicine, Toledo, USA;
Tel: 7346297458; E-mail: julia.shah@rockets.utoledo.edu 


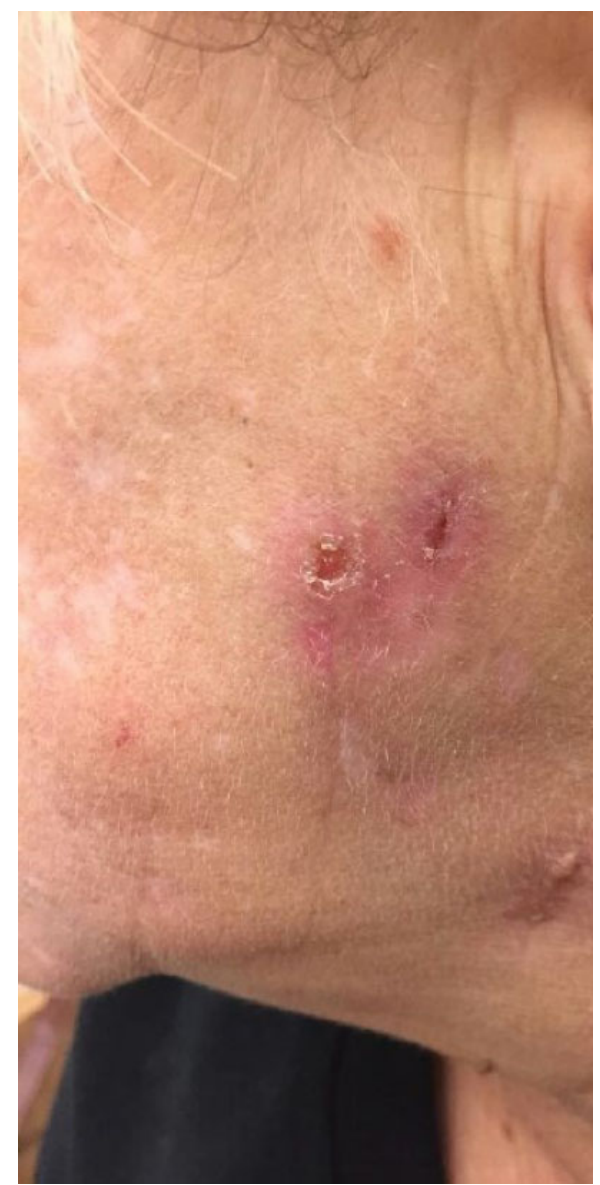

Fig. (1). left jaw.

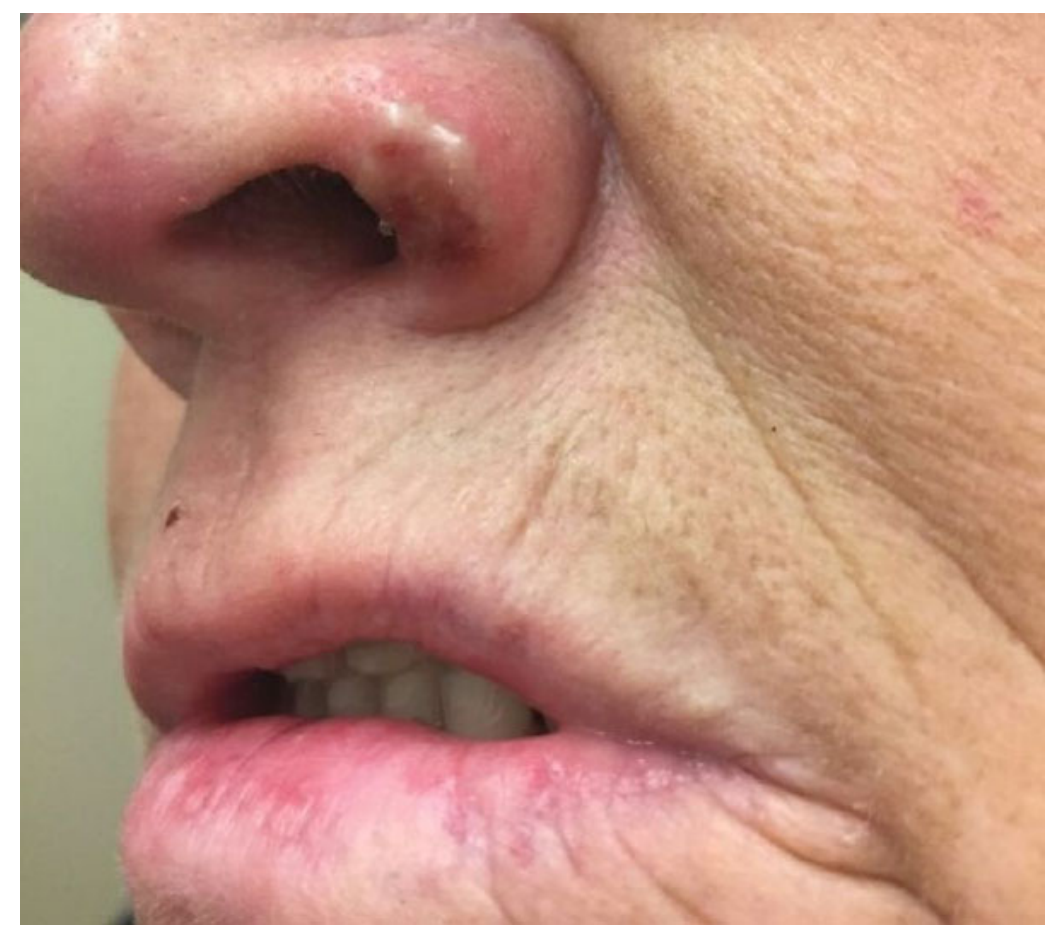

Fig. (2). left nostril. 


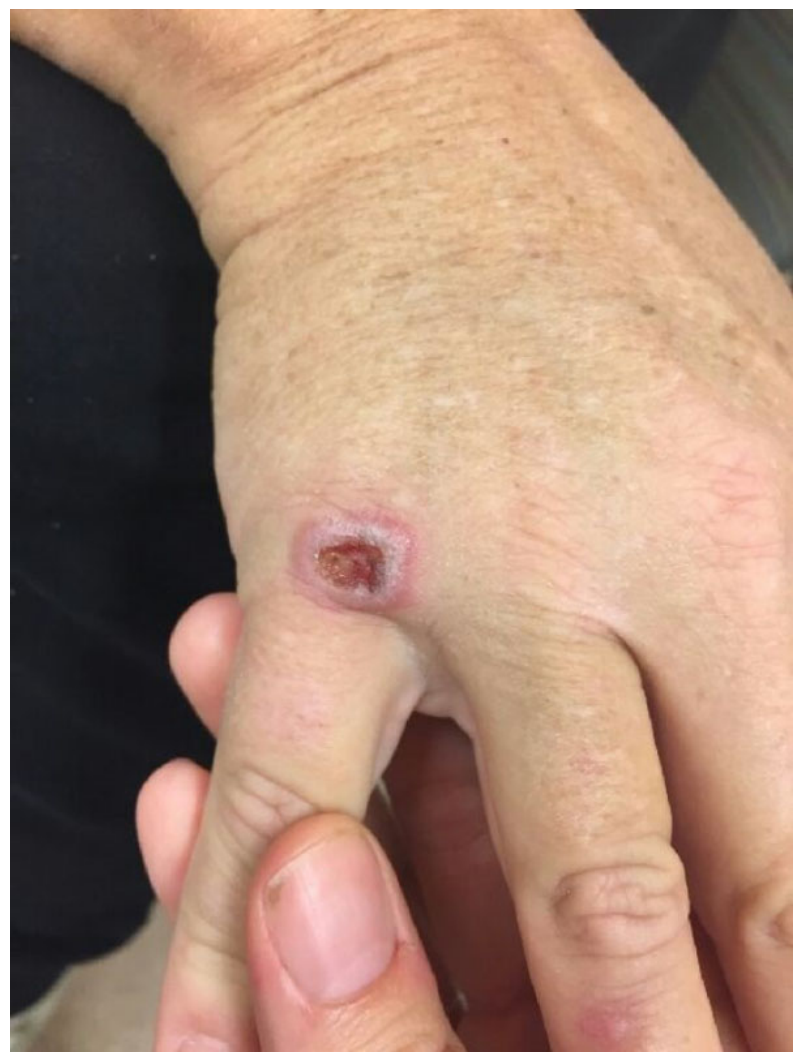

Fig. (3). Right fifth metacarpal phalangeal joint.

She was briefly lost to follow up and was seen 6 weeks later. The patient reported compliance with corticosteroid therapy and resolution of the lesions after course completion. On examination, resolution of the lesions on her hands and face was noted, with some mild scarring on her hand. She reported no new lesions at this time. She was instructed to follow up as needed and if any new ulcerations developed.

\section{DISCUSSION}

PG is characterized as a disorder of neutrophil chemotaxis [1]. The neutrophilic infiltration seen in PG is thought to be driven by the elevated levels of inflammatory mediators, such as IL-8 (neutrophil chemotactic factor), which are observed in the tissues [2]. The dense neutrophilic infiltrate present in our patient's lesion with a lack of infectious or malignant cause made PG the most likely diagnosis.

The Journal of the American Medical Association Dermatology recently published this consensus for the diagnostic criteria for PG: the presence of major criterion (a biopsy of an ulcer edge demonstrating a neutrophilic infiltrate) and the presence of at least four of the eight minor criteria (Fig. 4) ${ }^{1}$. Minor criteria include: (1) exclusion of infection; (2) pathergy; (3) history of inflammatory bowel disease or inflammatory arthritis; (4) history of papule, pustule, or vesicle ulcerating within 4 days of appearing; (5) peripheral erythema, undermining border, and tenderness at ulceration site; (6) multiple ulcerations, at least 1 on an anterior lower leg; (7) cribriform or "wrinkled paper" scar(s) at healed ulcer sites; and (8) decreased ulcer size within 1 month of initiating immunosuppressive medication(s) ${ }^{1}$. Our patient's case is consistent with the major criterion, and the first, second, third, fourth, fifth, and eighth aforementioned minor criteria.

The exact cause of PG is unknown, but patients tend to be between the age of 20-50 and males and females are equally affected [3]. Ulcerative PG, the most common variant, is typically found on the lower extremities [2]. The multi-focal ulcerative presentation above the waist is relatively rare but was shown in our patient. PG has also a tendency to cause pathergy, a phenomenon where minor trauma contributes to significant, non-healing skin damage [4, 5]. This is consistent with our patient's history of injuries preceding the formation of new PG lesions. 


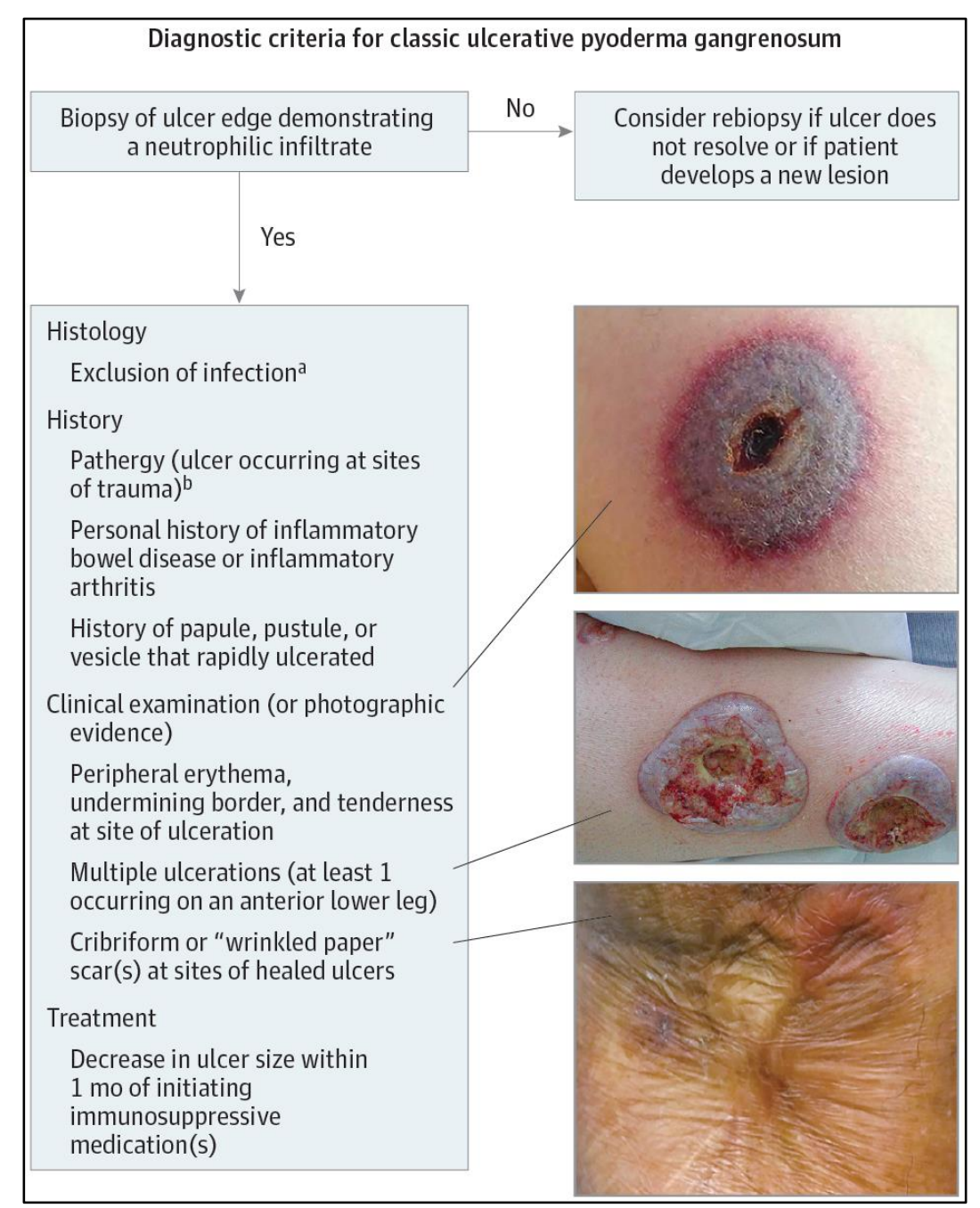

Fig. (4). JAMA Diagnostic Criteria of Ulcerative Pyoderma Gangrenosum A Delphi Consensus of International Experts .

PG has a well-known association with other neutrophilic and inflammatory disorders, including Inflammatory Bowel Disease (IBD), Rheumatoid Arthritis (RA), seronegative arthritis, autoimmune hepatitis [2, 6]. IBD is by far the most commonly observed. Some even classify seronegative arthritis and PG as extra-cutaneous manifestations of IBD. Our patient's history of seronegative arthritis may be associated with her skin disease. Affected patients exemplify a triad of IBD, PG, and seronegative arthritis concurrently [7]. PG has rarely been documented to occur with seronegative arthritis but without IBD - however, our patient is one such case [6].

PG has also been documented to occur as an adverse drug reaction. Paradoxically, these reactions are to the immunosuppressive drugs used to treat autoimmune conditions (e.g. IBD or RA) that occur with PG. A case report from the European Journal of Dermatology describes extensive ulcerative PG that developed in a psoriasis patient after 4 years of infliximab therapy [8]. Infliximab, an immunosuppressive agent, has also been reported to cause PG in at least two patients with ulcerative colitis, and one patient with RA [8].

Due to the rarity of PG, very few large-scale studies have been conducted comparing treatment options. First line treatment for limited PG, defined as involving $<5 \%$ of the body, is systemic or topical corticosteroids [2]. In a study conducted by the University of Nottingham, $47 \%$ of patients receiving $.75 \mathrm{mg} / \mathrm{kg} /$ day of prednisolone experienced resolution of PG at the end of a 6-month treatment period $[9,10]$. Patients in this study first began seeing improvement in symptoms after 6 weeks of oral prednisolone [10].

\section{CONCLUSION}

On average, most studies involving systemic steroid treatment for PG yield results after 6 weeks of treatment [9 11]. By contrast, our patient reported complete resolution of symptoms after a ten day treatment period with prednisone. 
The only other record of an abridged interval for steroid treatment is a case report of malignant pyoderma gangrenosum of the parotid gland with dexamethasone pulse therapy (100 mg dexamethasone in $500 \mathrm{ml}$ 5\% dextrose infused over 3-4 hours each day). The steroid treatment infusion occurred over three consecutive days and, even then, complete healing did not occur for 6 weeks [11]. By comparison, our patient's resolution of symptoms after just a 10-day treatment with oral prednisone is remarkable.

\section{CONSENT FOR PUBLICATION}

A written informed consent was obtained from the patient to produce the images. In accordance with the requirements of the HIPAA privacy act, the images have been edited to remove all direct and indirect patient identifiers.

\section{CONFLICT OF INTEREST}

The authors declare no conflict of interest, financial or otherwise.

\section{ACKNOWLEDGEMENTS}

Declared none.

\section{REFERENCES}

[1] Maverakis E, Ma C, Shinkai K, et al. Diagnostic criteria of ulcerative pyoderma gangrenosum: A delphi consensus of international experts. JAMA Dermatol 2018; 154(4): 461-6. [http://dx.doi.org/10.1001/jamadermatol.2017.5980] [PMID: 29450466]

[2] Alavi A, French LE, Davis MD, Brassard A, Kirsner RS. Pyoderma gangrenosum: An update on pathophysiology, diagnosis and treatment. Am J Clin Dermatol 2017; 18(3): 355-72.

[http://dx.doi.org/10.1007/s40257-017-0251-7] [PMID: 28224502]

[3] Ahronowitz I, Harp J, Shinkai K. Etiology and management of pyoderma gangrenosum: A comprehensive review. Am J Clin Dermatol 2012; 13(3): 191-211. [http://dx.doi.org/10.2165/11595240-000000000-00000] [PMID: 22356259]

[4] Zuo KJ, Fung E, Tredget EE, Lin AN. A systematic review of post-surgical pyoderma gangrenosum: Identification of risk factors and proposed management strategy. J Plast Reconstr Aesthet Surg 2015; 68(3): 295-303. [http://dx.doi.org/10.1016/j.bjps.2014.12.036] [PMID: 25589459]

[5] Patel DK, Locke M, Jarrett P. Pyoderma gangrenosum with pathergy: A potentially significant complication following breast reconstruction. J Plast Reconstr Aesthet Surg 2017; 70(7): 884-92.

[http://dx.doi.org/10.1016/j.bjps.2017.03.013] [PMID: 28476284]

[6] Olivieri I, Costa AM, Cantini F, Niccoli L, Marini R, Ferri S. Pyoderma gangrenosum in association with undifferentiated seronegative spondylarthropathy. Arthritis Rheum 1996; 39(6): 1062-5. [http://dx.doi.org/10.1002/art.1780390627] [PMID: 8651972]

[7] Card TR, Langan SM, Chu TP. Extra-Gastrointestinal manifestations of inflammatory bowel disease may be less common than previously reported. Dig Dis Sci 2016; 61(9): 2619-26. [http://dx.doi.org/10.1007/s10620-016-4195-1] [PMID: 27193564]

[8] Vestita M, Guida S, Mazzoccoli S, Loconsole F, Foti C. Late paradoxical development of pyoderma gangrenosum in a psoriasis patient treated with infliximab. Eur J Dermatol 2015; 25(3): 272-3.

[PMID: 25786592]

[9] Quist SR, Kraas L. Treatment options for pyoderma gangrenosum. Journal der Deutschen Dermatologischen Gesellschaft = Journal of the German Society of Dermatology. JDDG 2017; 15(1): 34-40.

[10] Ormerod AD, Thomas KS, Craig FE, et al. Comparison of the two most commonly used treatments for pyoderma gangrenosum: Results of the STOP GAP randomised controlled trial. BMJ 2015; 350: h2958. [http://dx.doi.org/10.1136/bmj.h2958] [PMID: 26071094]

[11] Ambooken B, Khader A, Muhammed K, Rajan U, Snigdha O. Malignant pyoderma gangrenosum eroding the parotid gland successfully treated with dexamethasone pulse therapy. Int J Dermatol 2014; 53(12): 1536-8. [http://dx.doi.org/10.1111/ijd.12519] [PMID: 25312614]

\section{(C) 2018 Shah et al.}

This is an open access article distributed under the terms of the Creative Commons Attribution 4.0 International Public License (CC-BY 4.0), a copy of which is available at: (https://creativecommons.org/licenses/by/4.0/legalcode). This license permits unrestricted use, distribution, and reproduction in any medium, provided the original author and source are credited. 